\title{
Prevalence of hypothyroidism in women with recurrent pregnancy loss in the first trimester in the Indian rural population
}

\author{
Verma $P^{1}$, Mathur $S^{2}$, Ranjan $V^{3}$ \\ ${ }^{1}$ Dr Pahula Verma, Resident, Department of Obstetrics \& Gynecology, ${ }^{2}$ Dr Sadhana Mathur, Professor, Department of \\ Obstetrics \& Gynecology, ${ }^{3}$ Dr. Vibhu Ranjan Sahni, Assistant Professor, Department of Oral \& Maxillofacial Surgery, all \\ authors are affiliated with NIMS Medical College \& Hospital, Jaipur, Rajasthan, India
}

Address for Correspondence: Dr Pahula Verma, Email: dr.pahulaverma@gmail.com

\begin{abstract}
Aim: To determine the frequency of hypothyroidism in women with recurrent pregnancy loss in first trimester in the Indian rural population. Study Design: The study included 50 women with one successful pregnancy and no history of miscarriages were selected as a control and total of 50 similar age group of pregnant women with recurrent pregnancy loss in gestational age up to $\leq 12$ weeks verified by pregnancy test or ultrasonography. Method: Levels of thyroid hormones T3, T4 and TSH were estimated in pregnant and non pregnant women with recurrent pregnancy loss and controls. Result: The hypothroidism was found to be more significant cause of recurrent pregnancy loss in women as comparative to the euthyroid women. Conclusions: The study demonstrates that hypothyroidism has a statistically significant relationship with recurrent pregnancy loss in the first trimester and suggests that diagnosis of hypothyroidism could help couples with recurrent pregnancy loss to have a successful outcome in subsequent pregnancies.
\end{abstract}

Keywords: Hypothyroidism, Recurrent pregnancy loss, Risk factor

\section{Introduction}

Recurrent pregnancy loss is historically defined as 3 consecutive pregnancy losses prior to 20 weeks from last menstrual period however newer guidelines from American Society of Reproductive Medicines have defined RPL as the loss of two or more pregnancies [1]. However according to recent guidelines the investigations should be started with 2 or more pregnancy loss. The pregnancy loss in the first trimester is 15 to $20 \%$ of clinically recognized pregnancies [2]. There are a few reports of prevalence of hypothyroidism during pregnancy from India with prevalence rates ranging from $4.8 \%$ to $11 \%$ [3]. Spontaneous loss is the most common complication of human pregnancy.

The majority of pregnancy losses occur prior to clinical detection, approximately $70 \%$ of human conception fails to achieve viability and an estimated $50 \%$ are lost before the first missed menstrual period [4]. Thyroid diseases are the commonest disorders affecting women of reproductive age group. In women incidence of hypothyroidism diagnosed before pregnancy is 1\% [5]. Hypothyroidism during pregnancy occurs in 1/1600-1/2000 (0.06-0.05)

Manuscript received: $24^{\text {th }}$ May 2016

Reviewed: $4^{\text {th }}$ June 2016

Author Corrected: $15^{\text {th }}$ June 2016

Accepted for Publication: $30^{\text {th }}$ June 2016 deliveries [6]. Probably the rarity of hypothyroidism in pregnancy is due to the fact that hypothyroid women have higher prevalence of anovulatory cycles and in case of conception they have higher rate of fetal loss in first trimester [7] [8]. The world wide prevalence of subclinical hypothyroidism ranges from 1-10\% [9]. Since these patients can develop overt hypothyroidism at rate of $5 \%$ per year it is important to identify these patients at risk [10]. Maternal hypothyroidism has been associated with increased fetal, neonatal losses and mental retardation with living euthyroid offsprings [11][12][13]. When subclinical hypothyroidism occurs in pregnancy, there is increased risk for miscarriage in both first and second trimester.

\section{Aim and Objective}

To determine the thyroid status in women with recurrent pregnancy losses in first trimester and controls to assess the relationship of hypothyroid with recurrent pregnancy losses.

\section{Materials and Methods}

The study was performed at the NIMS Medical college \& Hospital \& NIMS PHC Achrol, Jaipur. Study period was 
from June 2013 to June 2014. Total no of cases taken were 100 . Out of 100,50 patients were enrolled in study group and 50 in controls. The study group includes 50 non pregnant healthy women with two or more consecutive first trimester pregnancy loss. The control group includes 50 healthy matched women without a history of recurrent pregnancy loss. Inclusion criterias are non pregnant, normal uterine anatomy, on diabetic, APLA negative, TORCH negative. The exclusion criteria's are presently carrying pregnancy, acute or Chronic illness, receiving drugs(lithium, steroids) that could interfere with thyroid function tests, uterine anomalies, PCOS, abnormal husband semen analysis, APLA positive, TORCH positive. Detailed history of both partners followed by thorough clinical examination of all the women was taken at the institute. All the particulars have been taken and

\section{Original Research Article}

previous medical reproductive data were recorded in case sheets. Spouses of these women were not having any systemic diseases with complete semen analysis and normal sperm morphology. After taking written informed consent from the couples in the study $2 \mathrm{ml}$ blood was collected in a plain vial from each woman, serum was separated and store at $-20^{\circ} \mathrm{C}$ in small aliquots for hormonal investigations. The quantitative estimation of tri-iodothyronine and thyroxine hormone levels in serum samples was done by ELISA technique. The mean ${ }^{ \pm} \mathrm{SD}$ values of serum T3, T4 and TSH levels obtained in euthyroid and hypothyroid patients and controls. The quantitative estimation of TSH (thyroid stimulating hormone) is done by immune radiometric assay (IRMA). The normal range of $\mathrm{T} 3$ is $80-180 \mathrm{ng} / \mathrm{dl}$ and $\mathrm{T} 4$ 4.6$12 \mathrm{ug} / \mathrm{dl}$ and TSH $0.5-6.0 \mathrm{mIU} / \mathrm{ml}$.

\section{Results}

The mean maternal age of the 50 women with recurrent pregnancy loss was observed as $20.4^{+} 4$ years. The mean paternal age was observed $26^{+} 4$ years.

Table No.-1: Categorization of the study subjects $(n=100)$.

\begin{tabular}{|c|c|c|c|}
\hline Maternal age group & Study group & Control group & Average \\
\hline $20-25 \mathrm{yrs}$ & 18 & 20 & $22 \pm 1.5 \%$ \\
\hline $25-29 \mathrm{yrs}$ & 16 & 17 & $30.5 \pm 1.5 \%$ \\
\hline $30-34 \mathrm{yrs}$ & 14 & 10 & $31.2 \pm 1.5 \%$ \\
\hline 35 and above & 2 & 3 & $36 \pm 1.6 \%$ \\
\hline & $\mathbf{5 0}$ & $\mathbf{5 0}$ & \\
\hline
\end{tabular}

Table No.-2 Showing number of pregnancy losses.

\begin{tabular}{|c|c|c|}
\hline Study group & Pregnancy losses & Average \\
\hline 26 & 3 & $52 \%$ \\
\hline 24 & 2 & $48 \%$ \\
\hline
\end{tabular}

In the present study $26(52 \%)$ women experienced three or more pregnancy losses and 24(48\%) women experienced two losses.

Table No.-3: Mean + SD of serum T3 T4 and TSH levels in Hypothyroid and euthyroid women with recurrent pregnancy loss and controls.

\begin{tabular}{|c|c|c|c|}
\hline Thyroid status & Mean T3 level (ng/d) & Mean T4(mcg/dl) & $\begin{array}{c}\text { Mean TSH } \\
\text { level(mIU/ml) }\end{array}$ \\
\hline & Study group( $\mathrm{n}=50)$ & & $2.67 \pm 1.12$ \\
Euthyroid $(\mathrm{n}=46)$ & $120.49 \pm 19.8$ & $8.06 \pm 1.42$ & \\
Hypothyroid( $\mathrm{n}=4)$ & $55.5 \pm 8.55$ & $3.35 \pm 0.52$ & $2.29 \pm 1.27$ \\
\hline & Control group $(\mathrm{n}=50)$ & & 4.38 \\
\hline
\end{tabular}

In study group four $(8 \%)$ women out of 50 patients with recurrent pregnancy losses were suffering from hypothyroidism. In the control group one $(2 \%)$ woman was identified with hypothyroidism. 


\section{Original Research Article}

Statistical Analysis: The analysis was performed with the use of students two tailed t-test. The difference in the level of T3,T4 and TSH between euthyroid and hypothyroid in the patient group was statistically found to be highly significant $(p<0.001)$. There was only one hypothyroid case found in the control group, test of significance could not be carried out between euthyroid and hypothyroid women in controls. The result indicates that hypothyroidism is associated with recurrent pregnancy loss. In our study out of 15 out of 50 patients $(30 \%)$ with recurrent pregnancy loss had consanguineous marriages while in the control group 7 out of 50 patients $(14 \%)$ had such marriages. There was no significant correlation between consanguineous marriage and outcome of pregnancy $(\mathrm{p}=.0911)$ in our study.

\section{Discussion}

Thyroid hormones are essential for the substance of the developing fetus. Hence the treatment of a hypothyroid female is of prime importance. The fetus thyroid gland is not fully functional until after 12 weeks of pregnancy [2]. If the mother does not have sufficient thyroid hormones, she may be at increased risk of miscarriages. Thyroxin is essential for fetal neurodevelopment, maternal delivery of thyroxin to the fetus is essential early in gestation [14]. In pregnancy, iodide is lost through urine so the fetoplacental unit contributes to a state of relative iodine deficiency. Thus, pregnant ladies need additional iodine intake. A daily iodine intake of $250 \mu \mathrm{g}$ is recommended in pregnancy [15]. Some of the complications of hypothyroidism include decreased IQ u 14fetal death and miscarriage [16][17], visual motor deficiency in the offsprings [18] preterm delivery and post-partum thyroidits [19].

There is an increased risk of preeclampsia placental abruption, intra-uterine growth restriction, prematurity and intra-uterine fetal demise [14]. Therefore, the majority of the developed countries have national neonatal screening program [3]. The prevalence of hypothyroidism is more in Asian countries compare with the west; it varies from $2.5 \%$ from the West to $11 \%$ from India [20].

In our study the prevalence rate of hypothyroidism is $8 \%$ which is comparable to the study done by Upadhaya et al [14] showing the prevalence rate $13 \%$ (overt) and $31 \%$ (subclinical) hypothyroidism. The prevalence rate of hypothyroidism is also high in the studies done by Dinesh $\mathrm{K}$ Dhanwal et al [3] and AS Khalid et al [21] showing $14.3 \%$ and $11.45 \%$ respectively. A study done by Srinivas Rao et al [22] also demonstrated the prevalence rate of $20.1 \%$ which is quite high. KH Paikhambha et al [23] in his study showed high prevalence rate of hypothyroidism $23 \%$ which is also comparable with the results of our study.

Our study shows the mean TSH levels $29.84 \pm 21.13 \mathrm{mIU} / \mathrm{L}$ (hypothroid), $2.67 \pm 1.12 \mathrm{mIU} / \mathrm{L}$ which is similar with study done by the Dinesh K Dhanwal et al [3] showing mean TSH levels 3.16 (11.17)mIU/L. The results of our study is also comparable with the results of the study done by Amit R Baptre et al [7] and Alessandro Dal et al [24] showing mean TSH levels $10.024 \pm 4.13 \mathrm{mIU} / \mathrm{L}, 17.8 \pm 1.23 \mathrm{mIU} / \mathrm{L}$. An another study done by AS Khalid et al 21 also demonstrated high mean TSH levels $>2.5 \mathrm{mIU} / \mathrm{L}$ which is also similar to result of our study.

Majority of women are not sure that they are pregnant until four to six weeks and thus do not visit to doctor for their thyroid function test until their first trimester. Hence it is advisable to suggest the pregnant women to go for thyroid testing as soon as possible

\section{Conclusion}

Hence this study demonstrates that hypothyroidism has a statistically significant relationship with recurrent pregnancy losses in the first trimester. No significant relation between consanguineous marriages. Thyroid screening should be done to rule out subclinical hypothyroidism in recurrent pregnancy loss.

Funding: Nil, Conflict of interest: Nil Permission from IRB: Yes

\section{Reference}

1. Practice Committee of the American Society for Reproductive Medicine. Definitions of infertility and recurrent pregnancy loss. Fertil Steril 2008;89:1603.

2. Ramachandra R. V,Lakshmi .A ,Sadhnani M.D. Prevalence of Hypothyroidism in Recurrent pregnancy loss in first trimester. Indian Journal Med Sciences. september 2008;62(9)

3. Dhanwal D.K, Prasad S, Agarwal A.K, Dixit V. High prevalence of subclinical hypothyroidism during first trimester of pregnancy in North India. Indian J Endocrinol Metab. March 2013;17(2): 281-284.

4. Jonathen bereck, S. 33. In: Ruth blathi (ed.) Bereck and Novak's gynnecology 15th edt .: Lipinncott,Williams \& Wilkins; 2012. p. 1191.

5. Lovegreen J, Schust DJ. Maternal Hypothyroidism and Pregnancy Loss: Awaiting Firm Recommendations on 
Original Research Article

Testing and Treatment. Gynecology \& obstetrics (Sunnyvale, Calif). 2013; 3(1): 10.4172/ 2161-0932. 1000142. doi:10.4172/2161-0932.1000142.

6. Nuzhat, A, Reddy, P, Fernandez, E. Hypothyroidism in pregnancy: Is universal screening needed? J Obstet Gynecol India. November/ December 2006;56(6): 495-498.

7. Amit R Barapatre, Seema Vaidya. Study of thyroid proile in patients of recurrent abortions. Journal of Evolution of Medical and Dental Sciences 2013; Vol2, Issue 49, December 09; Page: 9614-9620.

8. Montoro, M.N. Hypothyroidism in pregnancy: Is universal screening needed?. Clin Obstet Gynecol. March 1997;40(1): 65-80.

9. Roti E, Minnelli R, Salvi M. Management of hypothyroidism in pregnant women. Journal Clinical Endocrinology \& Metabolism 1996; 81: 1679-82.

10. Pharoah P.O.D , Connolly K.J, Ekins R.P , Hardings ANN G. Maternal thyroid hormone levels in pregnancy and subsequent cognitive and motor performance of children. Clinical Endocrinology 1984; 21: 265-70

11. Brent GA. Maternal hypothyroidism: recognition and management. Thyroid.1999; 9:661-65

12. Kris Poppe, Brigitte Velkeniers, Daniel Glsnoer. Thyroid diseases \& Female reproduction. Clinical Endocrinology 2007, 66 (3): 309-21

13. WC Allan, J E Haddow, G E Palomaki, et al. Maternal thyroid deficiency and pregnancy complications. Journal of medical sciences 2000; 127-130

14. Upadhyaya TL, KC A, Paudel S. Prevalence and complications of Hypothyroidism during pregnancy in western Nepal. Nepal Journal of Medical sciences 2014;3(1):48-50.
15. Glinoer D.Thyroid disease during pregnancy in Werner and Ingbar's.9th ed. philadelphia: lippincot Williams and Wilkins:2004

16. Stagnaro-Green A ,Roman SH,Cobin RH.Detection of at risk pregnancy by means of highly sensitive assays for thyroid autoantibodies. JAMA 1990:264:1422-5

17. Allan WC,Haddow JE,Palomaki GE .Maternal thyroid deficiency and pregnancy complications:implication for population screening.J Med Screen 2000;7:127-30

18. Zoeller RT,Rovert J.Timing of thyroid hormone action in the developing brain:clinical observations and experimental findings.J Neuroendocrinol 2004;16:809-16

19. Nicholson WK, Robinson KA,Smallridge RC. Prevalance of postpartum thyroid dysfunction: A quantitave review. Thyroid 2006;16:573-82

20. Rao VR, Lakshmi A, Sadhnani MD. Prevalence of hypothyroidism in recurrent pregnancy loss in first trimester. Indian J Med Sci 2008;62:357

21.Khalid, A.S, Donoghue, K.O. Hypothyroidism and pregnancy loss: how significant is it?. Arch Dis Child Fetal Neonatal. 2010;95(1)

22. Rao S, Patibandla A. A Study to find out the Prevalence of Hypothyroidism among Pregnant Women Visiting ESI Hospital Sanathnagar Hyderabad. Gynecol Obstet (Sunnyvale) 2016; 6: 363.

23.Singh KP, Singh HA, Kamei H, Devi LM. Prevalence of Hypothyroidism among Pregnant Women in the SubMountain State of Manipur. Int J Sci Stud 2015;5 (5):143-146.

24. Alessandro dal lago, Elena vaquero, Patrizio pasqualetti . Prediction of early pregnancy maternal thyroid impairment in women affected with unexplained recurrent miscarriage. Human Reproduction. 2011;Vol26 (6): 1324-1330.

\section{How to cite this article?}

Verma P, Mathur S, Ranjan V.Prevalence of hypothyroidism in women with recurrent pregnancy loss in the first trimester in the Indian rural population. Obs Rev: J obstet Gynecol 2016;2(2):22-25. doi: 10.17511/joog.2016.i02.06. 